CHRONIQUE DE LA RECHERCHE

\title{
LIBERTINISME ET PHILOSOPHIE
}

\author{
Isabelle MOREAU
}

L'emploi des termes « libertin », « libertinisme », « libertinage », pour être attesté dans des textes de la fin du $\mathrm{XvI}^{\mathrm{e}}$ siècle et de la première moitié du XvII ${ }^{\mathrm{e}}$ siècle, n'offre pas de légitimité méthodologique a priori au chercheur désireux de trouver une catégorie endogène à l'histoire des idées. Ces dénominations, passablement floues en elles-mêmes, sont aujourd'hui en partie héritières de la tradition critique et fonctionnent, en tant que telles, sur des présupposés méthodologiques qui ne se recoupent pas toujours. On comprend qu'une telle question engage la définition de l'objet d'étude dans ses composantes comme dans ses manifestations historiques. Sans reprendre les différentes étapes d'un débat qui reste ouvert ${ }^{1}$, il convient de préciser simplement que de la définition, étroite ou élargie, du phénomène libertin dépendent ses bornes chronologiques ${ }^{2}$. À ce travail de délimitation et de périodisation éventuelle du corpus, s'ajoute une interrogation sur la nature de l'objet d'étude. Les termes «libertin » et «libertinage» ont perdu progressivement toute connotation philosophique et religieuse pour ne plus désigner, au XvIII ${ }^{\mathrm{e}}$ siècle, que l'extrême liberté et le dérèglement des mœurs. Constater cette évolution sémantique n'indique rien quant à la spécificité historique de cet objet. Faut-il distinguer, ou au contraire réunir dans une communauté d'analyse, un «libertinage » qui s'exprime dans les textes et un «libertinage de mœurs », autrement dit, des pratiques et des

1. Sur l'histoire de ces termes, voir Henri Busson, «Les noms des incrédules au XvI ${ }^{\mathrm{e}}$ siè- $^{-}$ cle », Bibliothèque d'humanisme et de Renaissance, t. XVI, 1954, p. 274-283. Pour le mot «libertin », voir l'ouvrage fondateur de Gerhard Schneider, Der Libertin. Zur Geistes- und Sozialgeschichte des Bürgertums im 16. und 17. Jahrhundert, Stuttgart, J. B. Metzler, 1970, ainsi que la contribution de Jean-Claude MARGolin, « Réflexions sur l'emploi du terme "libertin" au $\mathrm{xvI}^{\mathrm{e}}$ siècle », in Aspects du libertinisme au xvI siècle, actes du colloque international de Sommières, Paris, Vrin, 1974, p. 1-33. Voir aussi Jean WIRTH, « Libertins et épicuriens. Aspects de l'irréligion au $\mathrm{xvI}^{\mathrm{e}}$ siècle », Bibliothèque d'humanisme et de Renaissance, t. XXXIX, 1977, p. 601-627. Pour les sources chrétiennes de la notion et son emploi apologétique, voir l'ouvrage de Louise Godard De Donville, Le Libertin des origines à 1665. Un produit des apologètes, Paris/Seattle/Tübingen, Papers on French Seventeenth-Century Literature (Biblio 17), 1989. On se reportera, enfin, à l'article de Françoise Charles-Daubert, « Le libertinage érudit. Problèmes de définition », Libertinage et philosophie au XvII siècle, 1, 1996, p. 11-25.

2. Sur ces questions de périodisation, voir F. CHARLes-Daubert, « Libertinage et recherche contemporaine », XVII siècle, $\mathrm{n}^{\circ}$ spécial «Littérature, libertinage et philosophie au XVII ${ }^{\mathrm{e}}$ siècle », 149, oct.-déc. 1985 , p. 409-432, en part. p. 416 sq.

Revue de synthèse : $5^{\mathrm{e}}$ série, année 2005/1, p. 139-160. 
conduites de vie qui ressortissent de l'histoire des mentalités? Quant aux textes euxmêmes, faut-il établir une distinction entre la littérature et les poésies satiriques qui fleurissent au début du XVII ${ }^{e}$ siècle ${ }^{3}$ et une forme plus philosophique de libertinage : « libertinage érudit» ou encore «libertinisme», selon la terminologie retenue, au risque de dissoudre l'identité du phénomène libertin dans l'appellation générique (et anachronique) de «libre penseur»?

Si nous ne prétendons pas répondre à ces questions, nous entendons en revanche être claire sur ce qui fera l'objet de cet article : le mot de «libertinisme » entend désigner ici cet objet d'étude spécifique à l'histoire des idées, à la croisée des approches littéraire, philosophique et historique des textes : un objet textuel donc, caractérisé dans son rapport ambivalent à la philosophie ${ }^{4}$. Le libertinisme peut ainsi se définir comme l'expression littéraire et philosophique d'une mise en cause du savoir doxal en tant qu'il entraîne les croyances communes : l'hiatus instauré entre l'«esprit écarté » et le «vulgaire » recoupe l'opposition attendue entre privé et public. Il prend acte de l'ébranlement des certitudes consécutif aux découvertes scientifiques en physique, en astronomie ou en anthropologie, et use d'une raison et d'une méthode d'analyse où prévaut le versant critique et démystificateur, au détriment de toute construction systématique. Il est, enfin, l'expression d'un rapport antagoniste ou conflictuel à la religion : c'est une pensée irréligieuse - diverse en ses manifestations — qui entend se servir des systèmes philosophiques pour nourrir une polémique sceptique, antimétaphysique et antithéologique.

Parce que l'on ne peut réduire le phénomène libertin à une école de pensée ou à un corps de doctrine homogène, l'alliage des vocables «libertinisme » et "philosophie » ne va pas de soi. On ne peut que constater l'hétérogénéité des arguments employés ressortissant de systèmes philosophiques les plus divers - sans que, pour autant, cette absence d'unité au sein des sources inspiratrices permette d'en conclure à l'inanité philosophique de ce courant de pensée, encore moins à l'incohérence de ses «sectateurs ». Mais il faut souligner que le libertin, pour n'avoir pas adopté l'écriture systématique propre à une philosophie de concepts, ne se pense pas moins comme philosophe et revendique, comme pratique philosophique à part entière, un style d'écriture qui fait la part belle à la fiction (au sens le plus large de production de l'imagination). Autrement dit, le rapport du libertinisme à la philosophie ne se comprend qu'à la mode libertine, c'est-à-dire de biais, à l'aide de deux notions : notion de reprise, d'une part; de détournement, de l'autre.

3. Frédéric LachÈVRe, Bibliographie des Recueils collectifs de poésie publiés de 1577 à 1700, Paris, Honoré Champion, 1901-1906, 4 vol.; ID., Le Libertinage au xviI siècle. Disciples et successeurs de Théophile de Viau, Paris, Honoré Champion, 1909-1924, 11 vol., repr. Genève, Slatkine, 1968.

4. Si la distinction entre libertinage de mœurs et libertinage érudit a pu fournir à certains critiques une légitimité (morale) à leur objet d'étude, aucun jugement de valeur n'entre ici en ligne de compte. Le choix d'un vocable en -isme permet de résorber, en partie, les ambiguïtés constatées. En partie, car le mot « libertinisme », apparu brièvement dans l'apologétique protestante à la fin du $\mathrm{XVI}^{\mathrm{e}}$ siècle, à propos de sectes similaires à celles dénoncées par Calvin et Farel quelque cinquante ans plus tôt, est, en effet, utilisé par les spécialistes du XVI $\mathrm{e}^{\mathrm{e}}$ siècle pour désigner les divers courants hétérodoxes apparus dans la mouvance de ces «libertins spirituels » — équivoque fâcheuse : on comprendra qu'il est impératif de ne pas amalgamer hétérodoxie et irréligion lorsque l'on s'emploie à étudier la pensée antichrétienne au XVII ${ }^{\mathrm{e}}$ siècle. 
Reprise : le libertinisme au XVII ${ }^{\mathrm{e}}$ siècle, s'il ne fait pas école au sens académique du terme, renvoie en revanche à une certaine façon de se référer à une culture commune, de sélectionner au sein de la tradition certains arguments, plutôt que d'autres, pour se les approprier. Il faut alors bien distinguer ce qui relève de telle ou telle doctrine philosophique (comprise comme un argumentaire spécifique qui fait système), de la survivance de tel ou tel de ses arguments au sein de discours qui lui sont hétérogènes, parce que relevant d'une autre finalité.

Détournement : puisant largement dans la culture du paganisme gréco-latin ${ }^{5}$, les libertins projettent en retour leurs propres convictions sur les penseurs qu'ils utilisent. L'érudition libertine, sur ce plan, parce qu'elle sert à dissimuler et à diffuser des thèmes qu'il aurait été difficile de proposer autrement, se présente aussi comme une lecture et une interprétation nouvelle de l'Antiquité, même par rapport aux perspectives de la Renaissance. Inversement, les systèmes philosophiques qui s'élaborent au même moment ne peuvent pas ne pas tenir compte des problèmes récurrents soulevés par ces lectures irrévérencieuses - non tant pour les penseurs antiques, d'ailleurs, qu'en regard d'une tradition multiséculaire parvenue à intégrer l'Antiquité dans le christianisme. Enfin, les arguments et références ainsi détournés se retrouvent logiquement dans d'autres domaines de la philosophie, pénètrent la réflexion politique et viennent nourrir une production peut-être plus ouvertement « littéraire » - pensons aux romans de Cyrano de Bergerac — sans être moins philosophique. C'est dans ce va-et-vient que se comprend le mieux, à notre avis, le rapport complexe entretenu entre libertinisme et philosophie : un rapport tout de tensions, mais non d'indifférence.

Pour prendre la mesure d'un tel phénomène au sein de l'histoire des idées, une approche pluridisciplinaire n'est pas seulement recommandée, elle est une nécessité. On ne peut que se féliciter de constater la très réelle mise en œuvre, ces dernières années, d'une telle «circulation de matière », au sein même de la recherche ${ }^{6}$. En effet, le relatif désintérêt de la recherche française en matière de libertinisme et d'irréligion - désintérêt constaté en 1985 par Françoise Charles-Daubert, face à une Italie qui semblait avoir pris le relais des travaux de René Pintard et de Henri Busson — n'est plus tout à fait de mise ${ }^{7}$. La France tend à rattraper son retard — en

5. Pour un parcours critique de ces lectures libertines qui privilégient les doxographes et les historiens, comme Diogène Laërce et Diodore de Sicile, les moralistes, comme Plutarque et Lucien, qui s'ouvrent aussi à la pensée d'Épicure et de Sextus Empiricus et relisent Aristote, Cicéron ou Pline l'Ancien à la lumière de préoccupations nouvelles, on se reportera aux travaux de Tullio Gregory, Genèse de la raison classique de Charron à Descartes, Paris, Presses universitaires de France, 2000.

6. Nous avons choisi de nous concentrer sur les recherches anglo-saxonne, italienne et française, qui présentent, en ce domaine, des caractères particuliers.

7. Voir F. Charles-Daubert, art. cit. supra n. 2, p. 419. Sa bibliographie fait une large place aux ouvrages, articles, recueils d'articles et traductions produits par la recherche italienne. Pour les travaux pionniers qui ont donné à la recherche ses cadres d'étude, on se reportera aux ouvrages de Jean-Roger Charbonnel, La Pensée italienne au Xvi siècle et le courant libertin, Paris, Honoré Champion, 1917; H. Busson, La Pensée religieuse française de Charron à Pascal, Paris, Vrin, 1933; René PINTARD, Le Libertinage érudit dans la première moitié

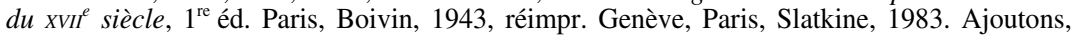
avec l'ouvrage de G. SchneIDER, op. cit. supra n. 1, les livres de John Stephenson SpINK: French Free Thought from Gassendi to Voltaire, Londres, The Athlone Press, 1960, trad. 
collaboration avec une Italie toujours aussi productive. Un rapide tableau des dernières initiatives universitaires, éditoriales et scientifiques devrait en témoigner.

Sur le plan universitaire, outre la récente mise au programme de l'agrégation d'un auteur comme Charles Sorel, divers séminaires sont sources de publications ponctuelles :

Séminaire «Espace public, censure et diffusion clandestine des idées aux XVII ${ }^{\mathrm{e}}$ et $\mathrm{XVIII}^{\mathrm{e}}$ siècles », à la bibliothèque Herzog August de Wolfenbüttel, sous la direction d'Edoardo Tortarolo, proposé dans le cadre de l'école d'été de Wolfenbüttel. À paraître, Epikureismus vom Humanismus bis zur Aufklärung. Recht, Moral und Politik, Herzog August Bibliothek, Wolfenbüttel, 23-24 novembre 2000, sous la direction de Gianni Paganini et d'Edoardo Tortarolo, Verceil, Università del Piemonte Orientale.

Séminaire "Littérature et philosophie au XvII ${ }^{\mathrm{e}}$ siècle», sous l'égide du Centre d'étude de la langue et de la littérature françaises des XVII et XVIII ${ }^{e}$ siècles, organisé par Emmanuel BuRY et Jean-Charles DARmon (depuis septembre 1996). Une première série de contributions a été publiée sous le titre "Littératures et philosophie au XVII ${ }^{e}$ siècle. Nouvelles perspectives », Papers on French Seventeenth-Century Literature, XXXV, 49, 1998. À paraître, le collectif Littérature et philosophie à l'âge classique, Paris, Honoré Champion, 2002, regroupant des contributions de divers participants du séminaire depuis 1997.

Séminaire « Histoire intellectuelle de l'Europe moderne. Secret et tromperie à l'époque moderne. Pratiques libertines », École des hautes études en sciences sociales-Centre de recherches historiques-Groupe de recherches interdisciplinaires sur l'histoire littéraire, sous la direction de Jean-Pierre CAvaiLlÉ.

Des colloques et journées d'études sont également organisés :

«Libertins et esprits forts du XVII ${ }^{\mathrm{e}}$ siècle. Quels modes de lecture? », journée annuelle d'études organisée par Pierre-François Moreau (ENS Lyon) et Antony McKenna (CNRS-UMR 5037), 26 avril 2001, École normale supérieure Lettres et Sciences humaines, Lyon, in Libertinage et philosophie au XVII siècle, 6, 2002.

«Le doute philosophique. Philosophie classique et littérature clandestine », Journée clandestine annuelle organisée par Geneviève ARTigas-Menant (UMR 8599) et Antony McKenna (UMR 5037), 18 mai 2001, université de Créteil Paris XII, La Lettre clandestine, 10, mai 2002.

«Descartes et les libertins », journées organisées par le Centre d'études cartésiennes, en collaboration avec le Centre d'études en rhétorique, philosophie et histoire des idées (CERPHI, ENS Fontenay) et le Centro di Studi su Descartes e il Seicento (université de Lecce), université Paris IV, 9 et 10 juin 2000.

franç. de Paul MeIEr, La Libre Pensée française de Gassendi à Voltaire, Paris, Éd. sociales, 1966, et de Giorgio SPINI, Ricerca dei libertini. La teoria dell'impostura delle religioni nel Seicento italiano, $1^{\text {re }}$ éd. Rome, 1950, Florence, La Nuova Italia, 1983. 
Sources antiques de l'irréligion moderne. Le relais italien ( $\mathrm{XV}^{e}$-XVII ${ }^{e}$ siècles), journées d'études des 3 et 4 décembre 1999, université de Toulouse-Le Mirail, actes publiés par Didier Foucault et Jean-Pierre Cavaillé, Toulouse, Presses de l'université de Toulouse-Le Mirail (coll. de l'E.C.R.I.T.), 6, juin 2001.

« Scepticisme et clandestinité », Dublin, sous la direction de Miguel BeníteZ et Gianni Paganini, et «Les libres penseurs et leurs adversaires », Dublin, sous la direction de James Dyвiкovski, tables rondes organisées dans le cadre du congrès des Lumières, parues sous le titre général Scepticisme, clandestinité et libre pensée, Paris, Honoré Champion, 2002.

«Le délit d'opinion à l'époque moderne. Du philosophe au colporteur», colloque international, automne 2004, Paris IV, sous la direction d'Antony McKenna, en collaboration avec Geneviève Artigas-Menant, université de SorbonneParis IV, CNRS URA 96.

« La communication manuscrite et l'évolution des idées à l'époque moderne », colloque international, septembre 2003, université de Genève, sous la direction de Maria-Cristina Pitassi, en collaboration avec Sarah Hutton, British Society for the History of Philosophy (non confirmé).

«Philosophie clandestine. Recherches récentes», table ronde, juillet 2003, Los Angeles, organisée dans le cadre du congrès des Lumières, sous la direction d'Antony McKenna et de Richard Popkin.

«Les Lumières radicales. La transformation philosophique entre 1650 et 1750 », colloque international, automne 2004, École normale supérieure Lettres et Sciences humaines, Lyon, sous la direction de Pierre-François Moreau, Catherine Secretan, Laurent Bove et Antony McKenna, en collaboration avec Jonathan IsRAËL, Princeton, USA.

À côté de ces manifestations, il faut noter l'émergence de collections universitaires spécialisées. L'édition de texte connaît, quant à elle, un regain de faveur, même si beaucoup reste à faire. Les principaux textes de la littérature libertine sont loin d'être tous disponibles et nombre de textes italiens attendent encore leur traduction.

\section{Publications périodiques :}

La Lettre clandestine, bulletin d'information sur la littérature philosophique clandestine à l'âge classique, sous la direction d'Olivier BLOCH et d'Antony McKenna, Paris, Presses universitaires de Paris-Sorbonne (parution annuelle depuis 1992, avec mise à jour bibliographique : 9 numéros parus).

Libertinage et philosophie au XVII siècle, sous la direction d'Antony McKenna et de Pierre-François Moreau, Saint-Étienne, Publications de l'université de SaintÉtienne (6 numéros parus). 
Éditions modernes, collections :

Libre pensée et littérature clandestine, sous la direction d'Antony McKenna, Oxford/Paris, Voltaire Foundation/Honoré Champion.

English deism and free-thought, sous la direction d'Antony McKennA, Oxford, Voltaire Foundation.

Philosophische Clandestina der deutschen Aufklärung, sous la direction de Winfried SchröDER, Stuttgart-Bad Cannstatt, Frommann-Holzboog.

Éditions de textes, liste indicative:

Libertins $d u$ XVII ${ }^{e}$ siècle, vol. I, édition établie, présentée et annotée par Jacques PRÉvот, Paris, Gallimard (Bibliothèque de la Pléiade), 1998. Textes édités : Théophile DE VIAU, [Théophile en procès]; Gabriel NAUdÉ, Apologie pour tous les grands personnages qui ont été faussement soupçonnés de magie; Tristan L'Hermite, Le Page disgracié; Pierre Gassendi, Traité de la philosophie d'Épicure, III partie : L'éthique ou la morale; Charles Dassoucy, Les Aventures de M. Dassoucy; Cyrano de Bergerac, L'Autre Monde. Les États et Empires de la Lune, Les États et Empires du Soleil; [anonyme], L'École des filles.

Theophrastus redivivus, edizione prima e critica del testo latino, a cura di Guido Canziani e Gianni Paganini, Florence, La Nuova Italia, 1981-1982, 2 vol., à présent diffusion Milan/Paris, Franco Angeli Editore/Vrin.

Cyrano de Bergerac, Euvres complètes. T. I : L'Autre Monde ou les États et Empires de la Lune; Les États et Empires du Soleil; Fragment de physique, édition critique, textes établis et commentés par Madeleine Alcover; t. II : Lettres. Entretiens pointus. Mazarinades, édition critique, textes établis et commentés par Luciano ERBA pour les Lettres et les Entretiens pointus et par Hubert CARrIER pour les Mazarinades; t. III : Théâtre, édition critique, textes établis et commentés par André Blanc, Paris, Honoré Champion, 2000-2001.

Jacques Vallée des BarReAux, La Vie et les auvres complètes de Jacques Vallée des Barreaux (1599-1673), par Marie-Françoise Baverel-Croissant, Paris, Honoré Champion, 2001.

Gabriel NAudÉ, Considérations politiques sur les coups d'État, édité par Louis Marin, Paris, Les Éditions de Paris, 1988 (précédé de L. Marin, Pour une théorie baroque de l'action politique, p. 7-65).

G. NAudÉ, Considerazioni politiche sui colpi di stato, précédé d'une «"Introduzione” a Gabriel Naudé » d'Alberto Piazzi (p. 3-93), Milan, Giuffrè, 1992.

G. NAudé, Considérations politiques sur les coups d'État, édité par Françoise Charles-Daubert, Hildesheim, Olms, 1993.

Samuel Sorbière, Relation d'un voyage en Angleterre, où sont touchées plusieurs choses, qui regardent l'estat des Sciences, \& de la Religion, \& autres matieres curieuses, fac-similé de l'édition de Cologne, Pierre Michel, 1666, présenté par Louis Roux, Saint-Étienne, Publications de l'université de Saint-Étienne, 1980. 
S. Sorbière, Discours sceptiques, édition critique établie et présentée par Sophie Gouverneur, Paris, Honoré Champion, 2002.

Giulio Cesare Vanini, Opere, a cura di Giovanni Papuli e Francesco Paolo RaIMONDI, Galatina, Congedo Ed., 1990.

Concernant le domaine particulier des manuscrits philosophiques clandestins, nous nous permettons de renvoyer aux bibliographies de la revue La Lettre clandestine, régulièrement mises à jour par Alain Mотнu ${ }^{8}$.

\section{BIBLIOGRAPHIE SÉLECTIVE}

$\mathrm{Au}$ vu des rapports complexes entretenus entre libertinisme et philosophie, nous avons choisi de présenter notre mise à jour bibliographique autour de quatre grands axes : libertinisme et politique, libertinisme et morale, libertinisme et religion, libertinisme et science. Cette méthode n'est pas sans présenter quelques inconvénients (risques de recoupement; ouvrages transversaux), mais elle nous a paru la plus à même d'offrir un instrument utile à la recherche. Le classement au sein de chaque rubrique se fait par ordre alphabétique.

Commençons d'emblée par le renvoi à quelques travaux fondateurs. L'ouvrage de René PINTARD constitue toujours une référence incontournable et une mine d'érudition pour le chercheur. On se reportera avec profit à sa mise au point méthodologique : «Les problèmes de l'histoire du libertinage, notes et réflexions », préface à la réédition du Libertinage érudit dans la première moitié $d u$ XVII ${ }^{e}$ siècle ${ }^{9}$. La synthèse de Françoise CHARLES-DAUBERT, «Libertinage et recherche contemporaine ${ }^{10}$ ", est un «état des recherches » des premières années du siècle aux années quatrevingt et une mise au point bibliographique de première importance — nous ne

8. Citons en outre : Le Matérialisme du XVIII siècle et la littérature clandestine, actes de la table ronde des 6 et 7 juin 1980, dir. Olivier BLoch, Paris, Vrin, 1982; De bonne main. La communication manuscrite au XVIII ${ }^{e}$ siècle, éd. François Moureau, Paris/Oxford, Universitas/ Voltaire Foundation, 1993; Filosofia e religione nella letteratura clandestina. Secoli XVII $e$ XVIII, dir. Guido Canziani, Milan, Franco Angeli, 1994; Miguel Benitez, La Face cachée des Lumières. Recherches sur les manuscrits philosophiques clandestins de l'âge classique, Paris/ Oxford, Universitas/Voltaire Foundation, 1996; Antony McKennA, « Les manuscrits philosophiques clandestins à l'âge classique. Bilan et perspectives de recherches ", XVII ${ }^{e}$ siècle, 192, juil.-sept. 1996, p. 523-535; ainsi que La Philosophie clandestine à l'âge classique, éd. Antony McKenna et Alain Mothu, Paris, Universitas, 1997. On se reportera aussi à l'article de Gianni Paganini, « Haupttendenzen der clandestinen Philosophie », in Grundriss der Geschichte der Philosophie. Bd I : Die Philosophie des 17. Jahrhunderts, hrsg. v. Jean-Pierre Schobinger. Teil 1: Allgemeine Themen, § 5, Bâle, Schwabe, 1998, p. 121-196 (avec une bibliographie très complète). Enfin, voir la Bibliographia clandestina, éd. Alain Mотнu : http://www.vc.unipmn.it/ mori/e-texts/bibclan.htm

9. R. Pintard, op. cit. supra n. 7, p. XIII-XLIII. Cette mise au point, « Les problèmes de l'histoire du libertinage », a paru également dans XVII siècle, 127, avr.-juin 1980, p. 131-161. Le numéro de cette revue est entièrement consacré au thème «Aspects et contours du libertinage ».

10. Voir art. cit. supra n. 2. 
reprendrons pas ses analyses, sauf renvoi ponctuel dans le cours de notre article. Du côté de la recherche italienne, l'article de Tullio GREGORY, «Il libertinismo della prima metà del Seicento, stato attuale degli studi e prospettive di ricerca ${ }^{11} »$, constitue une synthèse tout à fait suggestive. Il faut signaler, enfin, un panorama récent de la recherche établi par Martin MuLsow ${ }^{12}$, et la bibliographie établie par Sergio $\mathrm{ZOLI}^{13}$.

\section{Libertinisme et politique}

Un lieu commun de l'historiographie veut que la position des libertins - généralement taxés de conservatisme - se réduise à l'absolutisme, laissant leur entreprise critique s'arrêter au seuil du politique ${ }^{14}$. On assiste aujourd'hui à une réévaluation des données du problème. Le rapport des libertins à l'élaboration d'une pensée politique de la raison d'État est plus complexe qu'il n'y paraît et doit être envisagé au sein d'un processus global de laïcisation de la doctrine du droit et de l'État. Leur position se trouve, en effet, à la croisée de plusieurs traditions : celle du naturalisme italien de la Renaissance de source aristotélicienne et padouane, tel qu'il est attribué aux écrits de Cardan et Vanini; celle de Hobbes quant à la nécessité de l'obéissance à l'ordre politique; celle de Machiavel, via Charron et Vanini, quant aux moyens frauduleux d'obtenir cette obéissance. Elle apparaît irréductible à une simple défense et illustration de l'ordre monarchique et l'on assiste, de fait, au sein de la recherche actuelle, à la prise en compte croissante de son potentiel critique.

La remarque vaut pour le scepticisme. La polarisation de l'attention sur le lien scepticisme-religion s'est faite au détriment d'une étude de la rupture introduite entre morale et politique : le sceptique libertin, en déniant tout fondement théologique à l'État, lui refuse aussi une quelconque signification éthique. Une telle optique permet, d'une part, de créer un espace de liberté privé pour le sage, mais ouvre la voie, d'autre part, à une représentation tout extérieure du rapport du citoyen

11. Paru d'abord in Ricerche su letteratura libertina e letteratura clandestina nel Seicento, atti del convegno di studio di Genova, 30 ott.-1 nov. 1980, Florence, La Nuova Italia, 1981, cet article a été repris sous le titre «Le libertinisme dans la première moitié du XVII siècle », in T. Gregory, op. cit. supra n. 5, p. 13-62. Voir, également, les ouvrages de Domenico Bosco, Metamorfosi del "libertinage ». La "ragione esigente » e le sue ragioni, Milan, Vita e pensiero, 1982, et de Sergio Bertelli, Il Libertinismo in Europa, Milan/Naples, Ricciardo Ricciardi, 1980, «Presentazione », p. 3-24.

12. Martin Mulsow, «Cartesianismus, Libertinismus und historische Kritik», Philosophische Rundschau, Bd XLII, 1995, p. 297-314 (nous n'avons pu encore le consulter à ce jour).

13. Sergio Zoli, L'Europa libertina (secc. XVI-XVIII). Bibliografia generale, Florence, Nardini, 1997.

14. L'article de Lorenzo BiAnCHI, «Libertinisme et conservatisme politique. Le cas de Gabriel Naudé », voir infra, p. 147, constitue une bonne synthèse de la position traditionnelle sur le sujet : le conservatisme politique des libertins est indéniable et s'explique par la séparation instaurée entre le privé et le public, le sage et le vulgaire. Certes, mais que penser alors d'un libertin comme Cyrano? Sans même parler du rapport complexe de cet auteur à la Fronde, il manifeste, via Sejanus, un goût certain pour le régicide (et le parricide) et multiplie les épisodes politiques et les allusions à la « République » dans ses romans — qui n'ont rien d'utopique... 
à l'État, ce qui, en l'absence de lien contractuel, ne peut laisser intactes les assises du pouvoir étatique. Le rationalisme critique d'un Gabriel Naudé contribue tout autant à ruiner l'ancrage éthique et juridique du politique. En effet, la théorie machiavélienne de l'origine et de l'utilisation politique des religions est intimement liée, d'une part, à l'explication naturaliste d'événements considérés comme surnaturels, d'autre part, à la négation des valeurs qui assuraient, entre autres, la fondation éthique, théologique et rationnelle du politique. Au fondement transcendant des institutions se substitue une légitimité interne : l'unique souci de la paix et de la sûreté, tel qu'on le trouve chez Machiavel ou Spinoza, ou dans une certaine tradition aristotélicienne.

Cette réévaluation de la position libertine, où le conservatisme apparent (légitimé par la nécessité du maintien de l'ordre) va de pair avec une critique des prétentions du politique à chercher d'autre légitimité qu'en lui-même, n'est cependant qu'un des aspects d'une recherche qui mesure mieux aujourd'hui l'originalité de la dimension éthique propre à la position adoptée par le libertin face à la sphère publique - nous aborderons ce deuxième aspect dans la section suivante.

Ce secteur reste largement marqué par le dynamisme de la recherche italienne, forte d'une longue tradition de pensée en ce domaine. Il faut signaler d'emblée la grande bibliographie sur la raison d'État (1860-1993), parue dans Bollettino dell'Archivio della Ragione di Stato (Naples), vol. I, 1993, p. 15-92.

Anna Maria Battista, Alle origini del pensiero politico libertino. Montaigne e Charron, Milan, Istituto di Studi Storico-politici, Facolta di Scienze politiche, 11, 1966.

A. M. Battista, «Come giudicano la "politica" libertini e moralisti nella Francia del Seicento », in Il Libertinismo in Europa, sous la direction de Sergio BerTELLI, Milan/Naples, Ricciardo Ricciardi, 1980, p. 25-80.

Lorenzo BianchI, «Il libertinismo in Italia nel xvil secolo. Aspetti e problemi », Studi Storici (Rome, Gramsci Istituto), 3, 1984, p. 659-677.

L. BIANCHI, «Libertinisme et conservatisme politique. Le cas de Gabriel Naudé », Tijdschrift voor de Studie van de Verlichting en van het Vrije Denken, 14$15^{\mathrm{e}}$ Jaargang, 1986-1987, 4, 1987, Bruxelles, Vrije Universiteit, p. 385-404.

Olivier BLOCH, «La contestation libertine des normes et valeurs traditionnelles du Theophrastus redivivus au médecin Gaultier», in Ordre et contestation au temps des Classiques, édité par Roger DuchêNe et Pierre Ronzeaud, actes du $21^{\mathrm{e}}$ colloque du CMR 17, Marseille, juin 1991, Paris/Seattle/Tübingen, Papers on French Seventeenth-Century Literature (Biblio 17), 1992, t. II, p. 307-320.

Jean-Pierre Cavaillé, « Gabriel Naudé, Les Considérations politiques sur les coups d'État. Une simulation libertine du secret politique? », Libertinage et philosophie au xvII siècle, 2, 1997, p. 105-129.

J.-P. Cavaillé, Louis Machon. Une apologie pour Machiavel, Florence, Publication of the European University Institute, 4, 1998.

J.-P. CAvaillé, «La Mothe Le Vayer, libertinage et politique dans le Dialogue traitant de la politique sceptiquement ", in Materia Actuosa. Antiquité, âge classique, Lumières. Mélanges en l'honneur d'Olivier Bloch, Paris, Honoré Champion (Champion-Varia), 2000, p. 121-143. 
J.-P. Cavaillé, Dis/simulations. Religion, morale et politique au Xvil siècle. JulesCésar Vanini, François La Mothe Le Vayer, Gabriel Naudé, Louis Machon et Torquato Accetto, Paris, Honoré Champion, 2001.

Françoise Charles-DAuBert, «Le libertinage érudit et le problème du conservatisme politique », in L'État baroque, 1610-1652, sous la direction d'Henri Méchoulan, Paris, Vrin, 1985, p. 179-203.

F. Charles-Daubert, Les Libertins érudits en France au xvil siècle, Paris, Presses universitaires de France (Philosophies), 1998, en part., p. 48-65 et 92-96.

Vittor Ivo Comparato, «Il pensiero politico dei libertini », in Storia delle idee politiche economiche e sociali, sous la direction de Luigi Firpo. Vol. IV : L'Età moderna, Turin, Unione tipografico-editrice torinese, 1980, p. 95-164.

Jean-Charles DARmon, «Prudence politique et droit de propriété privée selon Bernier. Pour une analyse utilitariste de la décadence des Etats du Grand Mogol», Libertinage et philosophie au Xvil siècle, 3, 1999, p. 123-142. On se reportera à la section «Libertinisme et morale » (infra, p. 149-152) pour une bibliographie précise sur les thèmes de l'utilité et de l'intérêt, à la croisée des domaines politique et moral.

Carlo Dionisotti, Machiavellerie. Storia e fortuna di Machiavelli, Turin, Einaudi, 1980.

Eugenio Di RiEnzo, La Morte del Carnavale. Religione e impostura nella Francia del Cinquecento, Rome, Bulzoni, 1989.

Nannerl O. Keohane, Philosophy and the State in France. Renaissance to Enlightenment, Princeton, NJ, Princeton University Press, 1980, en part. la $2^{\mathrm{e}}$ partie sur le rapport entre libertinisme et absolutisme, «Interest and Prudence. The State and the Sage », p. 119-237.

Gianni Paganini, «Temps et histoire dans la pensée libertine », Archives de philosophie, t. XLIX, 1986, p. 583-602.

G. PAGaninI, «"Legislatores" et "impostores". Le Theophrastus redivivus et la thèse de l'imposture des religions au milieu du XvII siècle », in Sources antiques de l'irréligion moderne..., op. cit. supra p. 143, p. 181-218.

Adriano Pessina, «Virtù e storia nella riflessione politica di Gabriel Naudé », Ricerche su letteratura libertina e letteratura clandestina nel Seicento, op. cit. supra n. 11, p. 287-295.

Giuliano Procacci, «Un apologista machiavelliano del Grand Siècle: Louis Machon », in ID., Machiavelli nella cultura europea dell'età moderna, Rome, Bari, Laterza, 1995.

Michel Senellart, Les Arts de gouverner, Paris, Seuil, 1994, en part., p. 230-244 sur Naudé.

Giorgio SpINI, Ricerca dei libertini. La teoria dell'impostura delle religioni nel Seicento italiano, $1^{\text {re }}$ éd. Rome, 1950, $2^{\mathrm{e}}$ éd. revue et augmentée avec une importante mise à jour bibliographique, Florence, La Nuova Italia, 1983.

G. SPINI, «Alcuni appunti sui libertini italiani », in S. BerTelli, op. cit. supra n. 11, p. 117-124. 
Domenico Taranto, «Libertinismo e assolutismo, un rapporto critico? », Il Pensiero Politico (Florence), anno XXII, 2, 1989, p. 264-277.

D. Taranto, Pirronismo ed assolutismo nella Francia del'600. Studi sul pensiero politico dello scetticismo da Montaigne a Bayle (1580-1697), Milan, Franco Angeli, 1994.

Yves-Charles ZARKA, « Raison d'État, maximes d'État et coups d'État chez Gabriel Naudé », in Raison et déraison d'État, sous la direction de Y.-C. ZARKA, Paris, Presses universitaires de France, 1994, p. 151-169.

\section{Libertinisme et morale}

Le libertinisme, comme il a été dit, est inséparable d'un travail de relecture - travail de reprise et de détournement d'un fond commun constitué par les penseurs de l'Antiquité - qui entreprend de séparer définitivement christianisme et culture antique : cette dernière devient alors l'expression d'une raison parfaitement mondaine consciente de ses limites, le lieu où s'épanouit une sagesse tout humaine et une féconde réserve d'arguments contre les superstitions, les mythes et les traditions religieuses. La relecture de l'Antiquité a cet autre effet qu'elle transforme les doctrines philosophiques en recueils de lieux : la constitution de riches doxographies répond à l'exigence précise d'indiquer la diversité existant non seulement entre les différentes écoles philosophiques mais également au sein d'une nature humaine qui n'a plus rien d'homogène. Un tel procédé, en s'élevant contre les prétentions totalisantes de la raison dogmatique, conduit à un relativisme moral radical : d'une part, il combat efficacement la prétention humaine à se croire au centre de la création en dénonçant ses illusions anthropocentriques; d'autre part, il ruine l'argument du consentement universel, sur lequel s'appuie la tradition chrétienne pour prouver la théologie naturelle, l'immortalité de l'âme et l'existence de Dieu, et le remplace par le règne tout-puissant de la coutume et de l'opinion. Le relativisme critique conduit ainsi à une réduction en termes historiques des religions comme des lois éthiques qui n'ont, de fait, plus aucun fondement universel et transcendant.

$\mathrm{Au}$ croisement de ces diverses problématiques, Charron reste une référence incontournable: la Sagesse, qui connaît une vogue certaine au Xvir siècle, est lue comme la défense et la mise en œuvre d'une sagesse entièrement humaine et d'un relativisme qui vise, à travers la critique des lois et des valeurs universelles, le dogmatisme, les superstitions, les religions. Mais si la dissociation entre éthique et religion se répercute sur la sphère politique ${ }^{15}$, on comprendra qu'elle renvoie également à des enjeux anthropologiques et éthiques spécifiques. Le «naturalisme » qui soustend les thèses libertines, au sens le plus large de disposition intellectuelle portant à référer toute cause à la nature, dessine l'horizon fini dans lequel s'intègre tout comportement humain et propose les cadres d'une éthique antistoïcienne liée à la recherche du plaisir ou du «divertissement » dans les limites de ce monde.

C'est dans cette perspective qu'il faut lire les travaux les plus récents menés par tout un courant de la recherche sur les «passions libertines », sur leur statut et leur

15. Voir supra, p. 145. 
rôle tant sur le plan des mœurs que sur le plan philosophique. On se reportera notamment au volume Libertinage et philosophie au XvII siècle sur «Les passions libertines ${ }^{16}$. L'analyse des passions y apparaît comme un aspect essentiel de la « réhabilitation de la nature humaine », nature innocente appelée à s'épanouir dans l'exercice de toutes ses facultés. Ainsi mises en avant, elles sont tout à la fois le fondement de la vie sociale et de la vie politique; et ce qui en assure le fonctionnement - bon ou mauvais. Dévoyées, elles fondent les superstitions et suscitent la tyrannie. Ainsi l'une des théories qui donnaient de la religion une explication naturelle - en la rapportant à la crainte éprouvée par les premiers hommes - s'articule aussi à une réflexion d'ordre anthropologique : «La crainte serait la passion fondamentale à l'origine de la croyance aux dieux et à leur pouvoir ${ }^{17}$. » Nulle incompatibilité avec la théorie de l'imposture politique des religions : au contraire, puisqu'elle permet d'ancrer au cœur de la nature humaine cette force de la superstition qui assure aux politiques le caractère éminemment manipulable du «vulgaire». Mais si la réflexion anthropologique sur les passions apparaît comme ce qui permet d'assurer une légitimité indéniable à une politique de l'ordre (légitimité de la coercition pour contenir ces ferments de désordre que sont les passions) et possède une utilité pratique réelle (c'est parce qu'il en a connaissance que le bon politique sait gouverner le peuple), elle ouvre aussi sur la définition d'une éthique libertine qui ne condamne pas les passions mais sait en jouer et s'en amuser, voire s'en servir au nom de la recherche de l'utile - passion fondamentale appelée à un brillant avenir. Partisan de l'ordre public, le sage saura élaborer pour son propre compte une éthique sachant subordonner le bien public à son bien propre, une éthique visant à l'accomplissement privé, individuel, de ces mêmes passions que le politique était censé réprimer, ou tout au moins, contenir ${ }^{18}$.

La démarche est intéressante, d'autant qu'elle donne une certaine consistance aux positions éthiques assumées par les libertins, souvent taxés, en ce domaine, d'opportunisme moral, voire d'inconséquence philosophique. Elle rejoint une tendance récente qui accorde une attention accrue à l'anthropologie sceptique, centrée sur l'irrésolution, et à l'éthique sceptique qui en découle - parties autant méconnues qu'injustement décriées de cette philosophie. Si le libertinisme ne peut se réduire à la seule philosophie sceptique, on ne peut qu'applaudir à une revalorisation philosophique de cet objet d'étude - nous pensons ici, notamment, aux récents travaux de Sylvia Giocanti sur le scepticisme de La Mothe Le Vayer. On observerait une démarche similaire pour un autre grand courant philosophique qui alimente le libertinisme : l'épicurisme. Si, sous l'impact des travaux d'Olivier Bloch et de Gianni Paganini, notamment, la spécificité de la théorie politique de Gassendi commence à apparaître à l'horizon des recherches actuelles, il importe d'évaluer, dans ses « marges » — chez Cyrano de Bergerac ou Saint-Évremond, par exemple —, ce que les références au Jardin permettent de thématiser. Nous renvoyons ici aux travaux

16. Libertinage et philosophie au XVII siècle, 4, $2^{\mathrm{e}}$ partie, 2000.

17. Pierre-François Moreau, «La crainte a engendré les dieux », Libertinage et philosophie au хviI siècle, 4, 2000, p. 147-153.

18. On se reportera notamment à l'article de Sophie Gouverneur, « Samuel Sorbière, ou la réhabilitation libertine des passions », Libertinage et philosophie au XvII siècle, 4, 2000, p. 183-197. 
de Jean-Charles Darmon sur l'épicurisme et sa fortune philosophique et littéraire au $\mathrm{XVII}^{\mathrm{e}}$ siècle. En matière de réflexion politique ou morale, les notions d' «intérêt » et d' « utilitas » apparaissent centrales, à la croisée de relectures antiques et d'anthropologies nouvelles. Là encore, le libertinisme offre un regard original sur une notion qui n'est plus perçue comme un principe vicieux en soi mais comme une donnée inévitable et naturelle, constitutive d'une économie raisonnée des plaisirs, pour le sage, et partie intégrante de la société juridico-politique et des pratiques de pouvoir qu'elle induit.

On se reportera aux études suivantes :

Anna Maria BAtTistA, "Morale "privée" et utilitarisme en France au XVII siècle », Staatsräson. Studien zur Geschichte eines politischen Begriffs, herausgegeben von Roman Schnur, Berlin, Duncker und Humblot, 1975, p. 87-119.

Domenico Bosco, Morale della politica e individualisme nel Grand Siècle. Da Machiavelli a Bayle, Milan, C.U.S.L., 1984.

Jean-Charles DARmon, Philosophie épicurienne et littérature au xvII siècle en France. Études sur Gassendi, Cyrano, La Fontaine, Saint-Évremond, Paris, Presses universitaires de France (Perspectives littéraires), 1998.

J.-C. Darmon, «Le gassendisme frivole de Saint-Évremond», in Gassendi et l'Europe, 1592-1792, édité par Sylvia Murr, Paris, Vrin, 1997, p. 57-70.

J.-C. Darmon, «Le Jardin et la Loi. De l'utilité comme fondement du droit et du politique chez Pierre Gassendi », Littératures classiques, numéro spécial, «Droit et littérature », textes recueillis et présentés par Christian BIET, 40, automne 2000, p. 53-73.

J.-C. DARmon, «Variations néo-épicuriennes sur l'utilité des lois dans l'histoire d'un peuple. L'exemple de Saint-Évremond », in Le Droit a ses époques (de Pascal à Domat), université Blaise-Pascal, actes du colloque « Domat et sa postérité », Clermont-Ferrand, 19-21 septembre 1996, édité sous forme de CDRom, CERHAC, sous la direction de Dominique Descotes (UMR 5037).

J.-C. Darmon, «L'intérêt, la politique et l'histoire. Effets de sens néo-épicuriens », in Entre Baroque et Lumières. Saint-Évremond (1614-1703), actes du colloque de Cerisy, 25-27 septembre 1998, textes réunis par Suzanne Guellouz, Caen, Presses de l'université de Caen, 2000.

Sylvia Giocanti, « La perte du sens commun dans l'œuvre de La Mothe Le Vayer », Libertinage et philosophie au XvII siècle, 1, 1996, p. 27-52.

S. GiocantI, «La Mothe Le Vayer. Modes de diversion sceptique », Libertinage et philosophie au XVII siècle, 2, 1997, p. 33-48.

S. Giocanti, Penser l'irrésolution: Montaigne, Pascal, La Mothe Le Vayer. Trois itinéraires sceptiques, Paris, Honoré Champion, 2001.

Tullio Gregory, Etica e religione nella critica libertina, Naples, Guida, 1986, p. 11-70, réédité sous le titre «Éthique et religion dans la critique libertine », in Genèse de la raison classique de Charron à Descartes, op. cit. supra n. 5, p. 81-112. 
Gianni Paganini, «L'anthropologie naturaliste d'un esprit fort. Thèmes et problèmes pomponaciens dans le Theophrastus redivivus », XVII siècle, 149, octobre-décembre 1985, p. 349-378.

G. Paganini, Scepsi moderna. Interpretazioni dello scetticismo da Charron a Hume, Cosenza, Busento, 1991.

G. Paganini, "Libertins érudits. Des "libertins spirituels" aux "esprits forts" », in Dictionnaire d'éthique et de philosophie morale, sous la direction de Monique Canto-Sperber, Paris, Presses universitaires de France, 1996, p. 836-842.

\section{Libertinisme et religion}

L'extension polémique du mot «libertin» ne facilite pas une caractérisation claire de ce qu'il recouvre, en particulier dans son rapport à la religion. La recherche actuelle reste en partie tributaire des figures de «libertins » construites par les apologistes François Garasse et Marin Mersenne dans les années 1620: si la Doctrine curieuse des beaux esprits de ce temps (1623) du père Garasse constitue un tournant majeur parce qu'elle assure la fortune du mot, elle entérine, du même coup, son usage critique - et son flottement sémantique. Le mot se prête, de fait, à maints glissements de sens et en vient à s'appliquer à tout individu voulant se libérer de quelque contrainte que ce soit. Or, il ne nous semble pas que l'on puisse, sans risque, confondre affranchissement et insoumission, et encore moins hétérodoxie et libertinage au XVII siècle.

On rappellera d'abord que l'impiété (insoumission, voire agressivité envers la religion et absence de foi) est, pour les apologistes du début $\mathrm{du} \mathrm{XvII}^{\mathrm{e}}$ siècle, une composante essentielle du libertin — c'est d'ailleurs pour cette raison qu'il est «apprentif de l'athéisme ». Que l'on retrouve, par ailleurs, sous le «libertin » de l'apologétique, la figure du «persécuteur de la foi», patron et prototype, depuis Bède le Vénérable, de tous les ennemis de l'Église, n'implique pas qu'il faille dénier au référent toute réalité historique ${ }^{19}$.

Ensuite, si l'hétérodoxie se revendique comme seule orthodoxie relativement à une religion instituée perçue comme non légitime, l'irréligieux, au sens strict, se situe sur un autre plan. Nous avons ici l'une des pierres d'achoppement du discours critique, et pour nous, une composante essentielle du libertinisme au XVII siècle : la

19. La double étymologie, païenne et chrétienne, du mot «libertin » contribue au débat, selon que l'on met l'accent sur la dimension d'affranchissement, de liberté conquise, en se référant à l'étymologie latine et à son sens social et moral, ou que l'on rappelle derrière la figure du libertin celle de l'hérétique biblique. Voir les analyses de L. GodARD DE DonviLle, op. cit. supra n. 1, p. 23-26 et chap. I et II. Le grand apport de cette thèse réside dans l'éclairage apporté aux sources chrétiennes de la notion, sources qui nous paraissent essentielles en ce qu'elles recentrent la figure du libertin sur son impiété, au détriment d'une revendication de liberté trop floue pour être réellement pertinente — nous ne la suivrons pas, cependant, dans ses conclusions : si l'apologétique finit par construire un véritable personnage de libertin, il nous paraît surprenant d'en inférer la non-existence du phénomène historique, même complexe et divers en ses manifestations. 
possibilité d'une expression philosophique cohérente de positions antichrétiennes. Le débat, lancé dans les années quarante par Lucien Febvre ${ }^{20}$, en réponse à la thèse d'Abel Lefranc qui faisait de Rabelais un libre penseur moderne, porte sur la possibilité de l'existence d'une incroyance qui dépasse le stade de l'expression brute du «désespoir ». La question touche, bien évidemment, au rapport entre tradition aristotélicienne, philosophies antiques concurrentes et développement d'un athéisme doctrinal au sein de la culture occidentale. Febvre renvoie à plus tard l'émergence de systèmes de pensée cohérents, argumentés et athées, par manque d'outillage conceptuel, faisant ainsi bon marché de l'influence de certains systèmes philosophiques antiques, de la philosophie padouane et de l'averroïsme. Cependant, si affirmatif pour le $\mathrm{xvI}^{\mathrm{e}}$ siècle, du moins dans cet ouvrage, il se montre plus nuancé pour une époque postérieure. Et s'il prouve que le $\mathrm{xvI}_{\mathrm{e}}^{\mathrm{e}}$ siècle est « un siècle qui veut croire », Henri Busson, dès 1922, démontre que la Renaissance jette les bases du rationalisme et de l'athéisme en reprenant des penseurs de l'Antiquité païenne comme Lucien, Julien, Celse ou encore Averroè ${ }^{21}$.

Sans retracer l'historique de ces débats, qui se poursuivent encore aujourd'hui, on peut simplement signaler les travaux menés par Paganini sur cette somme de l'athéisme que constitue le Theophrastus redivivus. Ils ont largement démontré, à notre avis, l'importance de la tradition naturaliste, nourrie d'aristotélisme averroïste et de machiavélisme, pour le libertinisme au xvII ${ }^{e}$ siècle : «[...] le Theophrastus redivivus est là pour attester que, si ni Averroès, ni Pomponazzi, ni Cardan, ni Machiavel, ni Bodin, ni Vanini ne furent aucunement athées au sens rigoureux et complet du mot, ce fut cependant en lisant leurs œuvres qu'on pouvait quand même le devenir, dans une acception véritable et philosophique (et même en écrivant latin, quoi qu'en dise Febvre), et surtout sans qu'il fallût attendre l'avènement du cartésianisme ou la montée de la vague spinoziste ${ }^{22}$.»

À l'articulation du libertinisme et de l'irréligion, se trouve aussi l'hypothèse fidéiste, autre pomme de discorde parmi les critiques. Cette hypothèse, dont la fortune doit beaucoup à la grande synthèse offerte par Richard Popkin ${ }^{23}$, déplace le

20. Lucien Febvre, Le Problème de l'incroyance au XvI siècle. La religion de Rabelais,

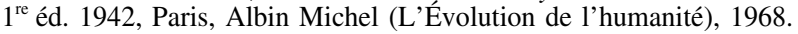

21. Voir H. Busson, Le Rationalisme dans la littérature française de la Renaissance (15331601), $1^{\text {re }}$ éd. 1922, Paris, Vrin, 1957. Il a largement contribué au débat en incluant, dans les rééditions de son ouvrage (éd. de 1957 et de 1971) une critique des thèses de L. Febvre. Voir aussi, entre autres, Jean WIRTH, art. cit. supra n. 1; François BerRIot, Athéismes et athéistes au XvI siècle en France, Paris, Cerf, 1984, 2 vol. Pour une évaluation très différente du problème, voir l'article de Paul Oskar Kristeller, «Le mythe de l'athéisme de la Renaissance », Bibliothèque d'humanisme et de Renaissance, t. XXXVII, 1975, p. 337-348 (trad. de « The myth of Renaissance atheism and the French tradition of free thought », Journal of the History of Philosphy, 1968, p. 233-343).

22. G. PAGAninI, "Legislatores" et "impostores". Le Theophrastus redivivus et la thèse de l'imposture des religions au milieu du XVII ${ }^{\mathrm{e}}$ siècle », in Sources antiques de l'irréligion moderne... , op. cit. supra p. 143, p. 181-218, citation p. 209.

23. Richard Popkin, The History of scepticism from Erasmus to Spinoza, $1^{\mathrm{re}}$ éd. The History of scepticism from Erasmus to Descartes, Assen, Van Gorcum \& C ${ }^{\circ}$, 1960, Berkeley, Los Angeles, Londres, University of California Press, 1979 pour l'éd. révisée et étendue retenue pour la trad. franç. par Christine Hivet, Histoire du scepticisme d'Érasme à Spinoza, Paris, Presses universitaires de France, 1995. 
débat sur le terrain du scepticisme : pour Popkin, le scepticisme développé à l'égard des arguments rationnels concernant les vérités de foi n'est pas une preuve d'incroyance; il sert plutôt à mettre la foi à l'abri de la discussion et donc de la discorde; c'est pourquoi l'on ne peut douter de la sincérité des professions de foi qui accompagnent ces reprises du scepticisme antique. Sans entrer dans les détails de la querelle, qu'il nous suffise d'en pointer ici les enjeux principaux. Le phénomène libertin $\mathrm{au} \mathrm{XVII}{ }^{\mathrm{e}}$ siècle ne peut en aucun cas se réduire à la seule expression d'une philosophie particulière, en l'occurrence le scepticisme moderne, mais on ne peut nier que cette dernière ne soit une des voies privilégiées du libertinisme. Sans remettre formellement en cause la possibilité d'un fidéisme sceptique " authentique ${ }^{24}$ », il est impératif d'en éprouver la compatibilité avec les présupposés théoriques et méthodologiques adoptés : il n'est pas sûr que l'alliance entre scepticisme et foi aille toujours de soi au $\mathrm{XVII}^{\mathrm{e}}$ siècle. Le fidéisme sceptique s'applique à distinguer la philosophie de la foi, tout en gardant le souci de penser l'une par rapport à l'autre : l'abdication de la raison se fait au profit de la foi, apte à mener le croyant à une certitude fiable. Rien de tel, en ce qui concerne un pyrrhonisme philosophique cohérent, qui fait de la difficulté de croire un obstacle insurmontable à la fermeté de la foi ${ }^{25}$. À prendre en compte l'environnement historique, et non plus conceptuel, ajoutons que le geste fidéiste ne revêt pas la même signification avant et après l'offensive de la Contre-Réforme - ce qui pouvait légitimement s'inscrire dans la mouvance d'un évangélisme d'inspiration paulinienne, relève ensuite, nous semble-t-il, de la provocation pure. Tout scepticisme moderne n'est pas à comprendre comme une propédeutique à la foi.

L'enjeu de ces différents débats est important et touche à la cohérence et à la consistance philosophique des auteurs concernés. La bibliographie qui suit présente les ouvrages ressortissant aux différents partis en présence dans la réflexion historiographique actuelle :

Recherches sur le XVII siècle. T.VI : Libertinage et incrédules (1665-1715), sous la direction d'André RoBINET, avec la collaboration de Pierre CLAIR, Paris, Éditions du CNRS, 1983.

Atheism from the Reformation to the Enlightenment, édite par David WoоттоN et Michael Hunter, Oxford, Clarendon Press, 1992.

Scepticism and irreligion in the 17th and 18th centuries, édité par Richard POPKIN et Arjo Vanderjagt, Leyde/New York/Cologne, Brill, 1993.

24. L'historien des idées, quoi qu'il en dise, ne peut juger de l'authenticité ou de la sincérité d'une posture relevant de la foi. Il ne peut qu'en évaluer la cohérence sur le plan des idées.

25. Le numéro 6, mars 1997, de la revue, Documents, archives de travail et arguments, éd. par le CERPHI (dir. P.-F. Moreau) École normale supérieure de Fontenay/Saint-Cloud, est consacré à l'Histoire du scepticisme de R. Popkin et comprend les articles suivants : Frédéric BRAHAMI, «L'articulation du scepticisme religieux et du scepticisme profane dans l'Histoire du scepticisme d'Érasme à Spinoza, de R. Popkin » (p. 3-11); Sylvia GiocantI, « Histoire du fidéisme, histoire du scepticisme?» (p. 13-28); Sophie Roux, "Quelques remarques sur la catégorie de scepticisme modéré » (p. 29-46). Voir aussi les mises au point critiques dans «Histoire du scepticisme. De Sextus Empiricus à Richard H. Popkin », Revue de synthèse, t. CXIX, $4^{e}$ sér., 2-3, avril-sept. 1998; et les analyses de S. Giocanti concernant le scepticisme de La Mothe Le Vayer, dans son ouvrage Penser l'irrésolution, op. cit. supra p. 151. Voir enfin le recueil de P.-F. Moreau, Le Scepticisme au Xvi et au XvII siècle, Paris, Albin Michel (Le Retour des philosophes antiques à l'âge classique), 2001. 
Heterodoxy, Spinozism, and free-thought in early eighteenth-century Europe. Studies on the "Traité des trois imposteurs", édité par Silvia BerTI, Françoise Charles-Daubert et Richard Popkin, Dordrecht/Boston/Londres, Kluwer, 1996.

Sources antiques de l'irréligion moderne. Le relais italien (XVI ${ }^{e}$-XVII ${ }^{e}$ siècles), op. cit. supra p. 143, notamment l'article de Jean-Pierre CAvaILLÉ, "L'historiographie de l'irréligion. Le relais italien», p. 3-13, et celui de Gianni PaganinI, «Le Theophrastus redivivus et la thèse de l'imposture des religions », p. 181-218, en particulier p. $206 s q$. , "Pour une histoire de l'athéisme à part entière ».

François Berriot, Athéismes et athéistes au xvI siècle en France, Lille, Le Cerf, 1984, 2 vol.

F. Berriot, Spiritualités, hétérodoxies et imaginaires. Études sur le Moyen Âge et la Renaissance, Saint-Étienne, Publications de l'université de Saint-Étienne, 1994, en particulier partie III, «De la spiritualité à l'hétérodoxie».

Frédéric Brahami, Le Travail du scepticisme. Montaigne, Bayle, Hume, Paris, Presses universitaires de France (Pratique théorique), 2001.

Michael J. Buckley, At the origins of modern atheism, New Haven, Londres, Yale University Press, 1987 (compte rendu critique par Alan Charles Kors, dans Eighteenth Century Studies, vol. XXII, 4, 1989, p. 614-617; voir aussi James E. ForCE, «The origins of modern atheism », Journal of the History of Ideas, L, 1989, p. 152-153).

Tullio Gregory, "Theophrastus redivivus ». Erudizione e ateismo nel Seicento, Naples, Morano, 1979. Cette étude contient l'interprétation historique la plus riche et la plus documentée de l'ouvrage.

Jean-Michel Gros, «Le masque du "scepticisme chrétien" chez La Mothe Le Vayer », Libertinage et philosophie au XVII siècle, 5, 2001.

Jonathan IsRaËL, The Radical Enlightenment. Philosophy and the making of modernity (1650-1750), Oxford, Oxford University Press, 2000.

Alan Charles Kors, Atheism in France, 1650-1729, Princeton, Princeton University Press, 1990-1993, 2 vol.

Hélène Ostrowiecki, Érudition et combat antireligieux au XVII siècle. Le cas du "Theophrastus redivivus », thèse de doctorat, Paris X-Nanterre, 1995.

H. Ostrowiecki, «La Bible des libertins », XVII siècle, 194, janv.-mars 1997, p. 43-56.

Gianni Paganini, « Bonheur, passions et intérêts. L'héritage des libertins », in L'État classique, édité par Henri Méchoulan et Joël Cornette, Paris, Vrin, 1996, p. 71-92.

G. Paganini, "Pyrrhonisme tout pur" ou "circoncis"? La dynamique du scepticisme chez La Mothe Le Vayer », Libertinage et philosophie au XVII siècle, 2, 1997, p. 7-31.

Raymond Trousson, Histoire de la libre pensée, des origines à 1789, Bruxelles, Espaces de libertés (Éditions du Centre d'Action laïque), 1993. 
David Wootton, «Lucien Febvre and the problem of early modern unbelief », The Journal of Modern History, vol. LX, 1988, p. 695-730, en particulier, p. 703707.

\section{Libertinisme et science}

On ne peut penser le développement des savoirs indépendamment du vivier historique et culturel qui le nourrit. Une attitude implicitement positiviste a cependant longtemps prévalu, qui a considéré le libertinisme à l'aune de son érudition, tout en assimilant plus ou moins ouvertement modernité et cartésianisme, révolution scientifique et science mécaniste. Cette approche a conduit à séparer l'étude de la science classique, ses grands développements et ses principaux hérauts (Galilée, Descartes, Huygens, Newton), des manifestations érudites d'un libertinisme «englué » dans sa tradition. Or, il nous paraît réducteur de ne voir dans l'érudition qu'un passéisme de mauvais aloi, sans prendre en compte sa très réelle dynamique gnoséologique. L'ajustement ne peut se faire qu'à condition d'accepter que l'engagement « rationaliste » du libertinisme puisse parcourir d'autres chemins que ceux de la raison dogmatique et se développe dans la lignée de l'enseignement sceptique comme méthode d'analyse historique et empirique consciente des limites de la raison humaine, cet « instrument utile dans un horizon humain où connaître c'est décrire historico stilo, sans tentations systématiques et totalisantes ${ }^{26} »$. La remarque vaut pour les manuscrits philosophiques clandestins au tournant du siècle : si le phénomène est relativement tardif (hormis le cas du Theophrastus), la science que ces écrits véhiculent n'est pas forcément la plus avancée. Et, s'ils ne sont pas tous anti-cartésiens, ils poursuivent en revanche, pour la plupart, la tâche entreprise par le courant libertin : rétablir des traditions matérialistes, déistes ou athées, reliant — par-delà la coupure cartésienne - sources anciennes et hétérodoxie moderne.

La pratique constante de la reprise, en dévoilant un autre visage de l'Antiquité et de la Renaissance, mettait à disposition des curieux tous les instruments d'une polémique contre des échelles de valeur sanctifiées. Elle contribuait aussi à tracer l'inventaire des « erreurs » humaines entérinées par la tradition, non sans retrouver, au passage, un certain nombre de schèmes explicatifs de l'homme et du monde incompatibles avec l'enseignement aristotélo-thomiste. L'originalité de la posture libertine dans le champ de la connaissance tient pour beaucoup de cette double orientation d'ébranlement des certitudes et de redécouverte, et de mise à l'épreuve, des textes et des systèmes antiques, renaissants ou contemporains. Au sein de l'héritage antique, l'attention se porte de façon privilégiée sur les doxographes, les moralistes, les historiens et les géographes de l'Antiquité tardive; on retrouve des auteurs oubliés, condamnés ou utilisés jusque-là différemment. Avec l'héritage renaissant, français et italien, c'est toute une tradition naturaliste qui se trouve réactivée, où confluent, de façon éclectique, thèmes aristotéliciens et références stoïciennes, épicuriennes, sceptiques. Nous avions évoqué, à propos de la possibilité d'un athéisme doctrinal conséquent, les débats concernant l'héritage italien : ils intéressent égale-

26. T. GREGORY, op. cit. supra n. 5, p. 97. 
ment le domaine épistémologique ${ }^{27}$. Vanini, sur ce plan, doit être considéré comme une médiation essentielle entre le naturalisme de la Renaissance et le libertinisme européen. Gregory notait en 1980 qu'il restait à évaluer précisément son œuvre dans ses composantes internes comme dans sa fortune ultérieure; les travaux se sont multipliés depuis, comblant en partie cette lacune.

S'il manque encore une véritable synthèse sur les choix de l'érudition libertine, un pas décisif a cependant été franchi à l'occasion de recherches menées sur la controverse qui opposa Descartes à Gassendi. Depuis quelques années, grâce aux travaux de Jean-Charles Darmon et de Sylvia Murr notamment, on mesure mieux l'importance de ce débat et son impact réel. Comme le souligne Pierre-François Moreau: «Derrière les deux protagonistes principaux du débat philosophique et scientifique au XVII ${ }^{\mathrm{e}}$ siècle se profilent des adversaires acharnés : d'un côté, les rationalistes chrétiens défendent une certaine idée de la philosophie, fondée sur la séparation des deux substances de l'esprit et du corps, et une certaine idée de l'apologétique, fondée sur la démonstration de l'existence de Dieu. De l'autre côté, les disciples de Gassendi, le défenseur d'un épicurisme moderne, qu'ils soient chrétiens comme Pascal, ou hétérodoxes comme Cyrano, insistent sur le rôle du corps dans la fabrication de nos idées et par conséquent sur le statut primordial de l'imagination $^{28}$. $\gg$ C'est toute une conception de la connaissance qui se dessine ici, où l'imagination joue un rôle essentiel comme voie d'exploration des possibles, afin de rendre compte au mieux des phénomènes observés : l'imagination est une des voies de la connaissance, elle est aussi première, préalable au travail de démystification. On comprendra que le lien entre libertinisme et science moderne ne soit alors ni direct ni univoque : les hypothèses scientifiques les plus modernes ne sont parfois retenues qu'en vertu des effets relativistes qu'elles induisent en matière de morale ou de savoir, ou parce qu'elles alimentent la crise des modèles anthropologiques traditionnels. Très souvent aussi, elles valent tout autant pour leur puissance évocatrice que pour leur potentiel scientifique : ainsi de l'hypothèse copernicienne qui fonctionne régulièrement, dans les textes libertins, à la fois comme un modèle critique et une matrice d'images.

Le relatif discrédit du libertinisme - souvent pensé en marge de la révolution scientifique - tient pour beaucoup à cette prééminence de l'imagination comme mode d'investigation du réel. Pourtant, la force et l'originalité de ce courant de pensée résident, d'une part, dans sa faculté de mettre en scène les « fables de la raison » pour mieux les confronter, d'autre part, dans le mode d'écriture qui en découle, plus littéraire que philosophique (au sens de construction systématique), mais non moins fécond, littérairement et philosophiquement.

«Aux sources du libertinage philosophique du xvII siècle. Giulio Cesare Vanini (1585-1619) », sous la direction de Jean-Pierre CAvaillé et de Didier Foucault, numéro spécial de Kairos, revue de la faculté de philosophie de l'université de Toulouse-Le Mirail, juin 1998.

27. Pour une position très critique sur le problème de l'héritage italien, voir les travaux de P. O. KRISTEller : art. cit. supra n. 21 ; «Between the Italian Renaissance and the French Enlightenment. Gabriel Naudé as an editor», Renaissance Quarterly, 1979, p. 41-71; Huit philosophes de la Renaissance italienne, Genève, Droz, 1975, trad. Anne Denis de Eight Philosophers of the Italian Renaissance, Stanford, CA, Stanford University Press, 1964.

28. P.-F. Moreau, « Avant-propos », Libertinage et philosophie au XVII siècle, 4, 2000. 
Révolution scientifique et libertinage, édité par Alain Mотнu et Antonella DeL Prete, Turnhout, Belgique, Brepols, 2000.

Materia actuosa. Mélanges en l'honneur d'Olivier Bloch, édité par Miguel BeNITEZ, Antony McKenna, Gianni Paganini et Jean Salem, Paris, Honoré Champion, 2000 .

Libertinage et philosophie au XVII siècle, $4,1^{\mathrm{re}}$ partie : "Gassendi et les gassendistes », 2000.

Giulio Cesare Vanini e il libertinismo, édité par Francesco Paolo RAIMONDI, atti del convegno di studi, Taurisano, 28-30 octobre 1999, Lecce, Congedo, 2000.

Lorenzo BiAnchi, Tradizione libertina e critica storica. Da Naudé a Bayle, Milan, Franco Angeli, 1988.

L. BIANCHI, «"Des novateurs modernes en la philosophie". Telesio tra eruditi e libertini nella Francia del Seicento », in Bernardino Telesio e la cultura napoletana, a cura di Raffaele SiRRI e Maurizio ToRrini, Naples, Guida, 1992, p. 373416.

L. Bianchi, Rinascimento e libertinismo. Studi su Gabriel Naudé, Naples, Bibliopolis, 1996 (le lecteur y trouvera une excellente mise au point bibliographique).

L. BianchI, « Renaissance et libertinage chez Gabriel Naudé », Libertinage et philosophie au XVII siècle, 2, 1997, p. 75-90.

Luca BiAnchi, L'Inizio dei tempi. Antichità et novità del mondo da Bonaventura a Newton, Florence, Olschki, 1987.

Carlo Borghero, La Certezza e la storia. Cartesianismo, pirronismo et conoscenza storica, Milan, Franco Angeli, 1983. Sur le rapport entre adoption d'un modèle non mathématique de connaissance et conception sceptique de l'histoire selon Gassendi et La Mothe Le Vayer.

Jean-Charles Darmon, Philosophie épicurienne et littérature, op. cit. supra p. 151.

J.-C. DARmon, « Gassendi contre Spinoza selon Bayle. Ricochets de la critique de l'Âme du monde », Archives de philosophie, juillet-septembre 1994, p. 523540.

J.-C. Darmon, «Gassendi et la "rhétorique" de Descartes », Papers on French Seventeenth-Century Literature, vol. XXXV, 49, 1998, p. 401-429.

Didier Foucault, Un philosophe libertin dans l'Europe baroque. Giulio Cesare Vanini (Taurisano, 1585-Toulouse, 1619), thèse de doctorat nouveau régime, sous la direction d'Yves CASTAN, université de Toulouse-Le Mirail, U.F.R. d'Histoire, 1997, 3 vol.

Tullio Gregory, «Aristotelismo e libertinismo », Giornale Critico della Filosofia Italiana, vol. LXI, 1982, p. 153-167, article traduit et réédité in Genèse de la raison classique de Charron à Descartes, op. cit. supra n. 5, «Aristotélisme et libertinisme », p. 63-80.

Thomas M. Lennon, The Battle of the Gods and Giants. The legacies of Descartes and Gassendi, 1655-1715, Princeton, NJ, Princeton University Press, 1993. 
Michel-Pierre Lerner, Tommaso Campanella en France au XVII siècle, Naples, Bibliopolis (Lezioni della Scuola di Studi Superiori di Napoli, Istituto Italiano per gli Studi Filosofici, 17), 1995.

Sylvia Murr, «Bernier et le gassendisme », Corpus, numéro spécial « Bernier et les gassendistes », 20-21, 1992, p. 115-135.

S. Murr, «Bernier et Gassendi. Une filiation déviationniste», in Gassendi et l'Europe, édité par S. Murr, actes du colloque international de Paris, «Gassendi et sa postérité (1592-1792)», Sorbonne, 6-10 octobre 1992, Paris, Vrin, 1997, p. 71-114.

Maria Emanuela Scribano, Da Descartes a Spinoza. Percorsi della teologia razionale nel Seicento, Milan, Franco Angeli, 1988.

Giorgio SpINI, Ricerca dei libertini, op. cit. supra p. 142, n. 7.

Sylvie Taussig, Les Lettres latines de Gassendi. Traduction et annotation, thèse dactylographiée, sous la direction de Jacques PrÉvot, université de Paris XNanterre, 1995-1996.

Sergio Zoli, Europa libertina tra Controriforma e Illuminismo. L' «Oriente » dei libertini e le origini dell'Illuminismo. Studi e ricerche, Bologne, Cappelli, 1989.

\section{LIBERTINISME ET RHÉTORIQUE LIBERTINE}

L'étude du libertinisme au XVII ${ }^{\mathrm{e}}$ siècle rencontre plusieurs difficultés qui tiennent à la diversité de ses cadres d'analyse. Il ne peut se caractériser par sa seule irréligion, notion trop vague pour être réellement pertinente lorsqu'elle est coupée de ses racines philosophiques. Il ne peut non plus, pour sa grande diversité et son approche a-systématique des questions philosophiques, être assimilé à un système de pensée particulier. Le rapport tendu qu'il entretient à la philosophie tient pour beaucoup de cet état de fait. Mais le libertinisme, c'est aussi une écriture, marquée par un certain nombre de procédés rhétoriques et stylistiques, et un mode d'expression philosophique à part entière. Là est son originalité et là est, à notre avis, un angle d'approche fécond, encore largement inexploré.

Qu'on nous permette de conclure cette chronique déjà longue par le renvoi à quelques travaux sur le sujet :

Jean-Pierre Cavaillé, «L'art d'écrire des philosophes », Critique, 631, décembre 1999, p. 969-980, compte rendu des ouvrages de Gianluca MoRI, Bayle philosophe, Paris, Honoré Champion, 1999, et de Laurent JAFFro, Éthique de la communication et art d'écrire. Shaftesbury et les Lumières anglaises, Paris, Presses universitaires de France, 1998.

Jean-Charles DARMon, «Écriture polémique et libertinages de la pointe. Autour des Lettres de Cyrano de Bergerac », in L'Écriture polémique, textes réunis par Michel Murat et Gilles DeCLERQ, Paris/Genève, Champion/Slatkine, à paraître. 
Joan DeJean, Libertine strategies. Freedom and the novel in seventeeth-century France, Colombus, Ohio State University Press, 1981.

Sophie Gouverneur, «Le Mascurat. Un exemple d'écriture libertine », Libertinage et philosophie au XvII siècle, 2, 1997, p. 131-143.

Isabelle Moreau, «Pour un protocole de lecture libertine. Pour ou contre les sorciers de Cyrano de Bergerac », La Lettre clandestine, 9, 2000, p. 314-327.

I. Moreau, «Gabriel Naudé. Une apologie de la prudence en matière de lecture », Libertinage et philosophie au XVII siècle, 6, 2002.

Isabelle MOREAu, Université Jean-Monnet, Saint-Étienne Institut Claude-Longeon, MRASH 35, rue du 11-Novembre 42023 Saint-Étienne Cedex 2 (novembre 2001). 